\title{
Multi-vessel disease and CZT SPECT. Comparison with coronary angiography
}

\author{
Nikant Sabharwal, BSc, BM BCh, DM, FRCP, and Avijit Lahiri, MBBS, MSc, \\ MRCP, FACC, FESC ${ }^{\mathrm{b}, \mathrm{c}}$ \\ ${ }^{a}$ Oxford Heart Centre, John Radcliffe Hospital, Oxford, United Kingdom \\ ${ }^{b}$ Clinical Imaging and Research Centre, Wellington Hospital, London, United Kingdom \\ ${ }^{\mathrm{c}}$ Middlesex University, London, United Kingdom
}

Received Dec 29, 2015; accepted Dec 29, 2015

doi: 10.1007/s12350-016-0432-4

\section{See related article, pp. 687-695}

The thorny issue of multi-vessel balanced ischemia raises it head again. One of the greatest fears in Nuclear Cardiology is the fact that we might miss a "balanced", multi-vessel ischemia situation and call the perfusion scan normal. Underidentification and treatment of these patients is an ongoing problem. ${ }^{1}$ Conversely, underdiagnosing the extent of coronary lesions with SPECT once a patient has already been referred to angiography, due to the severity of identified ischemia, is forgivable. If we identify single-vessel disease but the patient has two separate coronary lesions, then the principle of "no harm" can be ascribed apart from possibly underdetecting the total ischemic burden.

The problem of globally reduced relative perfusion has generated a whole raft of indices that help the reporter to identify a "normal" scan from a homogenously hypo-perfused but balanced ischemic myocardium. Transient ischemic dilatation, a drop in stress LVEF, RV prominence, lung uptake with thallium 201 and more recently coronary calcification have all been proposed as potential indicators of multi-vessel ischemia. The frequency of missed balanced multi-vessel ischemia is possibly lower than what we realize when an experienced reader is reporting the scans. ${ }^{2}$

The greatest assumption is that the invasive luminogram (aka invasive coronary angiogram) is the

Reprint requests: Nikant Sabharwal, BSc, BM BCh, DM, FRCP, Oxford Heart Centre, John Radcliffe Hospital, Oxford, United Kingdom; nikant.sabharwal@ouh.nhs.uk

J Nucl Cardiol 2017;24:696-7.

1071-3581/\$34.00

Copyright (C) 2016 American Society of Nuclear Cardiology. "gold standard". As a result, anatomical-based investigations will then tend to be more "accurate" that functional tests. ${ }^{3}$ The discussion regarding perfusion versus anatomical assessment is advanced and mature. It is well recognized that diffuse $\mathrm{CAD}$ can produce ischemic defects in the absence of discrete stenosis. For this reason, FFR evaluation of "moderate" CAD is considered reasonable in all but the most obviously stenosed vessel. ${ }^{4}$ Ideally, we should be moving away from the anatomy/perfusion debate and looking at patient-focussed events such as improvement in symptoms and cardiac event rates. However, in the "real world" the practicalities of providing medical care can sometimes be very different to the published clinical trial literature.

The authors of this paper should be congratulated on taking on this task with a defined set of parameters that apply a "real world" situation. Patients with chest pain and either an intermediate likelihood of IHD or known CAD were subjected to both $\mathrm{Tc}^{99 \mathrm{~m}}$-tetrofosmin CZT SPECT and coronary angiography (invasive or CT). Bicycle stress was the predominant stress method employed. A low-dose one-day stress-rest protocol was utilized. Results are stratified according to semi-quantitative SSS and SDS evaluation of perfusion by two experienced reporters.

The results confirm that on a per-patient basis, a SSS $>7$ ( $>10 \%$ of LV myocardium) showed a sensitivity of $74 \%(95 \%$ CI $70 \%$ to $79 \%)$ and a specificity of $83 \%$ (95\% CI $80 \%$ to $89 \%$ ) in detecting $>70 \%$ coronary stenosis lesions on quantitative angiographic criteria. In $16 \%$ of patients, MPI did not show significant perfusion abnormalities despite the presence of angiographic CAD, while in $6 \%$ it was positive in the absence of obstructive coronary lesions (more frequent in diabetics and those with multi-vessel disease). The false positive results occurred in older patients and those with 
confirmed prior myocardial infarction. CZT SPECT in this study was equally as good as confirming single-, double- or triple-vessel coronary disease.

We are not told if there was much disagreement between reporters or if they were blinded to angiographic data. It is also not clear whether any weighting to regional ischemia was applied. For example, a patient with a single severe proximal LAD stenosis would be expected to have a large ischemic burden. However, a patient with several moderate stenoses in a multi-vessel pattern may have a smaller ischemic burden. ${ }^{5}$ Have we underestimated the ischemia or overestimated the importance of the angiographic disease or both?

So where do we go from here?

Can we conclude that normal CZT SPECT perfusion excludes multi-vessel CAD? Definitely not, as the sensitivity and specificity from this study do not provide us with sufficient evidence to support this approach. However, the patient is in a prognostically lower risk category. That may not be enough to provide succor to the patient or interventional cardiologist.

Are we getting better at identifying multi-vessel disease? Probably, but problems remain regarding the reference standard of luminal stenosis. CZT cameras will ultimately improve our confidence and accuracy and therefore improve our ability to treat patients. A final sobering thought is a trawl through the archives and a look at some of the original literature. ${ }^{6}$ On the twentieth anniversary of this seminal $\mathrm{Tc}^{99 \mathrm{~m}}$-tetrofosmin study using planar imaging, we have a similar sensitivity, when compared to coronary angiography, to this publication using CZT SPECT.
Ultimately treating symptoms and reducing cardiac events should be the mainstay of cardiology. Our role as cardiac imagers will be to utilize our available tools and use them appropriately. CZT SPECT is definitely a useful modality in this regard.

\section{References}

1. Melikian N, De Bondt P, Tonino P, et al. Fractional flow reserve and myocardial perfusion imaging in patients with angiographic multivessel coronary artery disease. JACC Cardiovasc Interv 2010;3(3):307-14. doi:10.1016/j.jcin.2009.12.010.

2. Yokota S, Ottervanger JP, Mouden M, et al. Prevalence, location, and extent of significant coronary artery disease in patients with normal myocardial perfusion imaging. J Nucl Cardiol 2014;21:28490 .

3. Arbab-Zadeh A, Di Carli MF, Cerci R, et al. Accuracy of computed tomographic angiography and single-photon emission computed tomography-acquired myocardial perfusion imaging for the diagnosis of coronary artery disease. Circ Cardiovasc Imaging 2015;8(10):e003533. doi:10.1161/CIRCIMAGING.115.003533.

4. Van Nunen LX, et al. Fractional flow reserve versus angiography for guidance of PCI in patients with multivessel coronary artery disease (FAME): 5-year follow-up of a randomised controlled trial. Lancet 2015. doi:10.1016/S0140-6736(15)00057-4.

5. Tamarappoo BK, Gutstein A, Cheng VY, et al. Assessment of the relationship between stenosis severity and distribution of coronary artery stenoses on multislice computed tomographic angiography and myocardial ischemia detected by single photon emission computed tomography. J Nucl Cardiol 2010;17(5):791-802. doi: 10.1007/s12350-010-9230-6.

6. Zaret B, Rigo P, Wackers F, et al. Myocardial perfusion imaging with $99 \mathrm{mTc}$ tetrofosmin. Comparison to $201 \mathrm{Tl}$ imaging and coronary angiography in a phase III multicenter trial. Circulation 1995;91:313-9. doi:10.1161/01.CIR.91.2.313. 